\title{
Multimodal imaging: an evaluation of univariate and multivariate methods for simultaneous EEG/fMRI.
}

Citation for published version (APA):

de Martino, F., Valente, G., de Borst, A. W., Esposito, F., Roebroeck, A. F., Goebel, R. W., \& Formisano, E. (2010). Multimodal imaging: an evaluation of univariate and multivariate methods for simultaneous EEG/fMRI. Magnetic Resonance Imaging, 28(8), 1104-1112. https://doi.org/10.1016/j.mri.2009.12.026

Document status and date:

Published: 01/01/2010

DOI:

10.1016/j.mri.2009.12.026

Document Version:

Publisher's PDF, also known as Version of record

Document license:

Taverne

Please check the document version of this publication:

- A submitted manuscript is the version of the article upon submission and before peer-review. There can be important differences between the submitted version and the official published version of record.

People interested in the research are advised to contact the author for the final version of the publication, or visit the DOI to the publisher's website.

- The final author version and the galley proof are versions of the publication after peer review.

- The final published version features the final layout of the paper including the volume, issue and page numbers.

Link to publication

\footnotetext{
General rights rights.

- You may freely distribute the URL identifying the publication in the public portal. please follow below link for the End User Agreement:

www.umlib.nl/taverne-license

Take down policy

If you believe that this document breaches copyright please contact us at:

repository@maastrichtuniversity.nl

providing details and we will investigate your claim.
}

Copyright and moral rights for the publications made accessible in the public portal are retained by the authors and/or other copyright owners and it is a condition of accessing publications that users recognise and abide by the legal requirements associated with these

- Users may download and print one copy of any publication from the public portal for the purpose of private study or research.

- You may not further distribute the material or use it for any profit-making activity or commercial gain

If the publication is distributed under the terms of Article $25 \mathrm{fa}$ of the Dutch Copyright Act, indicated by the "Taverne" license above, 


\title{
Multimodal imaging: an evaluation of univariate and multivariate methods for simultaneous EEG/fMRI
}

\author{
Federico De Martino ${ }^{\mathrm{a}, *}$, Giancarlo Valente ${ }^{\mathrm{a}}$, Aline W. de Borst ${ }^{\mathrm{a}, \mathrm{c}}$, Fabrizio Esposito ${ }^{\mathrm{a}, \mathrm{b}}$, \\ Alard Roebroeck $^{\mathrm{a}}$, Rainer Goebel ${ }^{\mathrm{a}}$, Elia Formisano ${ }^{\mathrm{a}}$ \\ ${ }^{a}$ Department of Cognitive Neuroscience, Faculty of Psychology and Neuroscience, Maastricht University, 6200 MD Maastricht, The Netherlands \\ ${ }^{\mathrm{b}}$ Department of Neuroscience, University of Naples "Federico II", 80125 Naples, Italy \\ ${ }^{\mathrm{c}}$ Department of Neuroscience, University of Pisa, Pisa, Italy \\ Received 11 October 2009; revised 17 December 2009; accepted 21 December 2009
}

\begin{abstract}
The combination of electroencephalography (EEG) and functional magnetic resonance imaging (fMRI) has been proposed as a tool to study brain dynamics with both high temporal and high spatial resolution. Multimodal imaging techniques rely on the assumption of a common neuronal source for the different recorded signals. In order to maximally exploit the combination of these techniques, one needs to understand the coupling (i.e., the relation) between electroencephalographic (EEG) and fMRI blood oxygen level-dependent (BOLD) signals. Recently, simultaneous EEG-fMRI measurements have been used to investigate the relation between the two signals. Previous attempts at the analysis of simultaneous EEG-fMRI data reported significant correlations between regional BOLD activations and modulation of both eventrelated potential (ERP) and oscillatory EEG power, mostly in the alpha but also in other frequency bands.

Beyond the correlation of the two measured brain signals, the relevant issue we address here is the ability of predicting the signal in one modality using information from the other modality. Using multivariate machine learning-based regression, we show how it is possible to predict EEG power oscillations from simultaneously acquired fMRI data during an eyes-open/eyes-closed task using either the original channels or the underlying cortically distributed sources as the relevant EEG signal for the analysis of multimodal data.
\end{abstract}

(C) 2010 Elsevier Inc. All rights reserved.

Keywords: Multimodal imaging; Univariate and multivariate methods; EEG/fMRI

\section{Introduction}

The complementary nature of electroencephalography (EEG; high temporal resolution and low spatial resolution) and functional magnetic resonance imaging (fMRI; high spatial resolution and low temporal resolution) makes their combination appealing for investigating human brain dynamics [1-6].

When combining fMRI with electrophysiological recordings, the limitations posed by the indirect nature of the blood oxygen level-dependent (BOLD) signal (e.g., the difficulty in distinguishing between excitation and inhibition) have to be considered $[6,7]$. BOLD signal changes were shown to relate to local field potentials (and to a lesser degree to spike

* Corresponding author. Tel.: +31 433884532; fax: +31 433884125.

E-mail address: f.demartino@maastrichtuniversity.nl (F. De Martino). rates) using simultaneous invasive electrophysiological recording and fMRI in monkeys [8]. In humans, intracortical recordings and BOLD imaging acquired in different populations of subjects (i.e., nonsimultaneous) have been used for a similar purpose [9]. Noninvasive multimodal imaging in humans uses measures of electrophysiological changes on the scalp. Thus, this technique faces additional challenges to the fruitful study of brain dynamics. First, the measured scalp electrical activity reflects more global changes when compared to intracranial recordings and thus might represent information differently. Second, the coupling between electrical signals measured on the scalp and fMRI BOLD signals has to be understood.

In the last few years, several techniques have been proposed to combine brain signals as measured by EEG and fMRI into one analytical framework. These methods range from the separate analysis of the data and subsequent juxtaposition of the results to truly integrated methods $[1,2]$. 
The combination of brain signals acquired with different imaging modalities relies on the assumption of common neuronal sources generating the different signals. In the case of EEG and fMRI this results in assuming that blood oxygen level changes depicted in the fMRI data and electrical activity at the level of the scalp as measured by EEG are generated from the same neuronal activity. Truly integrated analysis of simultaneous EEG-fMRI makes this assumption explicit in the model while simple juxtaposition verifies this assumption post hoc, when interpreting the results. The superiority of a truly integrated method has been recently demonstrated from an information-theoretic point of view [10], showing that the joint distribution of responses from the two data sets is more informative than the single modalities.

Integrated analysis of multimodal data can be divided into two categories of methods [3]: (1) fMRI-constrained EEG analysis (equivalent current dipole estimates and distributed current dipole estimates); (2) EEG-constrained fMRI analysis (fMRI correlates of EEG power modulations, trialby-trial coupling).

In the context of multimodal imaging, the development of simultaneous EEG-fMRI measurements offers several advantages over separate session recordings, despite the degraded EEG data quality. Simultaneous measurements guarantee identical sensory stimulation, perception and behavior, and also provide a unique way to study how ongoing fluctuations of brain signals can reflect anticipatory signals that interact with, and modify, the processing of sensory events [5].

Simultaneous recordings and integrated analysis techniques have been used to study the coupling between superficial EEG power modulations and the fMRI BOLD signal at rest. Correlating the fMRI time courses on a voxelby-voxel basis to the power oscillations in specific frequency bands extracted from the EEG measurements, several studies have reported regional BOLD changes as being coupled with the EEG power modulations in different frequency bands [9,11-23]. However, such a 'massively univariate' approach does not take into account the intrinsic multivariate nature of the fMRI data and thus can be suboptimal in detecting relations to EEG power oscillations that are weak and spatially distributed in the fMRI data.

Multivariate analysis of the fMRI data [independent component analysis (ICA)] can be used to highlight networks of functionally connected areas. The correlation between such networks and the EEG power modulations in different frequency bands has also been used to describe the coupling of the two measured signals [24]. The nature of ICA allows extracting functionally connected networks, from fMRI data, without making use of a stringent hypothesis on their temporal evolution (i.e., 'blindly'). Thus, different independent components (ICs) can be coupled to the same EEG rhythm, thereby leaving unresolved the problem of finding the unique (if present) fMRI network that explains most of the information in a specific EEG band.
Multiway partial least squares (N-PLS) [25] has been used to estimate the combination of voxel BOLD signals that correlates best with the EEG and the combination of EEG signals that correlates best with the BOLD signal in a unified data-driven solution that does not rely on the a priori definition of the relevant EEG bands.

The use of correlation as the measure of the coupling between surface EEG and fMRI is reliable in explaining the relations between the available data sets (e.g., correlation between the time course of an IC of fMRI data with EEG power spectrum modulation). However, in order to estimate a pattern (i.e., multivariate model) in the fMRI associated with a specific EEG signal (thus not using 'blind' models of the fMRI data [24] or EEG data [25]), the use of a correlation-based approach is highly prone to overfitting (i.e., explaining EEG modulations with random multivariate noise in the fMRI). In order to avoid overfitting, the generalization to different data sets (i.e., predictive modeling) can be used instead of the correlation to validate the estimated relation between fMRI patterns and EEG signals.

Recently, predictive models have been introduced to investigate the relation between multivariate fMRI BOLD signals and a continuous experimental variable [Pittsburgh Brain Activity Interpretation Competition (PBAIC) 2006 and 2007, http://www.lrdc.pitt.edu/ebc/PBAIC.html] $[26,27]$. These methods are particularly suited to the analysis of fMRI data given the typical dimensionality of the problem (number of voxels $\gg$ number of samples). Beyond the simple correlation, these methods allow, after a learning phase, the prediction of the experimental variable (i.e., stimulus, behavior) by exploiting the multivariate information present in the data.

In this article, we introduce the use of multivariate machine learning-based regression to the multimodal analysis of the coupling between two brain signals. More specifically, we evaluate the possibility of predicting EEG power oscillations from simultaneously acquired BOLD fMRI.

Previous studies that investigated the relation between EEG power oscillations and fMRI BOLD in humans mostly used resting paradigms [11-14,17-23] and have reported high intersubject variability for the resulting regional correlations [16] and a high level of dependence in the correlations between different frequency bands in the EEG signal and in the BOLD signal (i.e., similar fMRI maps obtained from the correlation of alpha and beta oscillations with the BOLD signal) [22]. In this context, the multimodal analysis has been restricted to the EEG signal coming from one electrode [19], a group of electrodes [11-14,16-18,21, $22,24]$, the weighted average of electrodes estimated using ICA [15,20] or global field synchronization [23].

In order to validate the use of multivariate regression for multimodal imaging, here we test the ability of this method to predict EEG power oscillations in a paradigm that consists of alternating blocks of rest with eyes open and eyes closed [15]. We expect this paradigm, known to induce high alpha 
power modulations in the occipital cortex, to reduce intersubject variability and interaction among frequency bands when compared to simple resting with eyes open or eyes closed.

In a first analysis, we focus on the prediction of EEG power modulations estimated on the time course of selected temporal ICs $[15,20]$ and compare prediction accuracies obtained with multivariate regression schemes to the accuracy obtained by previously introduced correlation (univariate and multivariate)-based approaches. In a second analysis, we use cortically constrained distributed source modeling [28-30] to estimate which cortical regions are associated with the highest modulation in EEG power between eyes open and eyes closed periods and predict from the fMRI signal the EEG power modulations at the level of the cortical sources.

We show that multivariate regression is considerably more accurate than previously introduced correlation-based approaches in predicting the subjective alpha oscillations induced by opening and closing the eyes at the level of scalp distribution. We also show similar prediction performances when the focus is shifted from scalp distribution to cortical sources.

\section{Methods}

\subsection{General description of the approach}

Fig. 1 illustrates the main steps of our approach. The original EEG data are preprocessed in order to remove the artefacts induced by the magnetic resonance imaging (MRI)

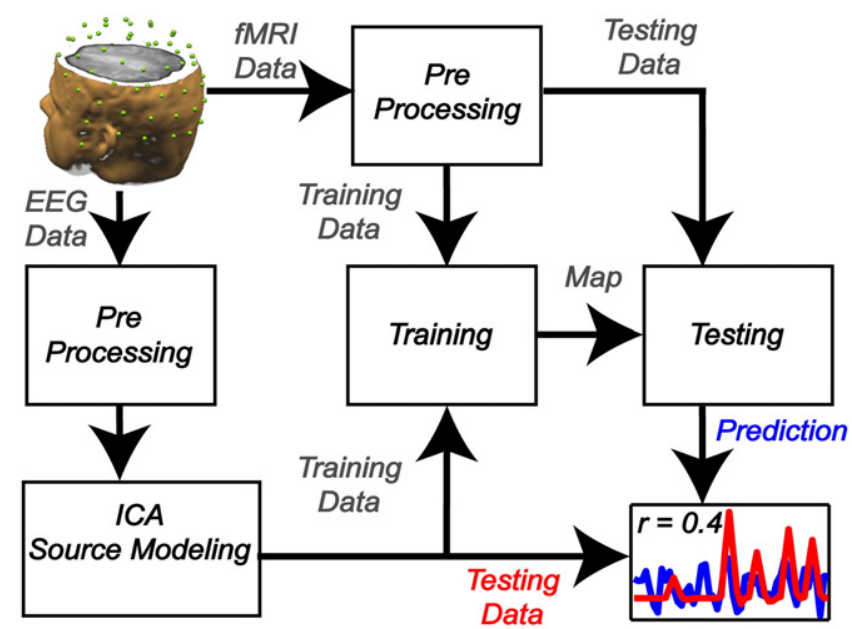

Fig. 1. General description of the proposed multivariate approach for the prediction of EEG features (e.g., power modulation in a specific band) from fMRI patterns. After conventional preprocessing, both the EEG and fMRI data are divided into training and testing sets. The link between fMRI patterns and EEG features is learned (making use of RR or RMVs) using the first set (i.e., training). After the training phase a map depicting the contribution of each voxel to the learned coupling is obtained. The model is evaluated on the basis of the correlation between the predicted and real EEG features of the second data set (i.e., testing). environment (gradient artefact and ballistocardiogram (BCG) artefact [31-33]). The preprocessed data are then decomposed using temporal ICA (tICA) [34], and relevant components are selected based on their scalp distribution and power spectrum. Sources of EEG activity on the reconstructed cortical surfaces of the subject can be estimated using distributed source-modeling techniques [28-30]. After time frequency analysis, predictors are then computed by averaging the power oscillations of the selected IC, or of the cortical sources in a region (i.e., surface patch) of interest, in a selected frequency window (e.g., alpha). The predictors are finally convolved with a canonical hemodynamic response function (HRF) [35] and resampled at the time resolution of the fMRI data.

Both data sets (i.e., the preprocessed fMRI time series and EEG-based predictors) are then divided into training and testing. The training data set is used to learn the coupling between the fMRI and the EEG oscillations in the selected frequency band using relevance vector machine (RVM) and ridge regression (RR). A map depicting the contribution of each voxel to the learned coupling is obtained. The test data are used to assess the validity of the learned coupling by means of the prediction accuracy (i.e., the correlation between the predicted and real EEG oscillations).

\subsection{Multivariate regression of fMRI time series}

In the context of simultaneous EEG-fMRI recordings, the fMRI time series, represented by the $N \times V$ matrix $\mathbf{X}$ ( $N$ being the number of volumes and $V$ the number of voxels), and the EEG power modulations in a specific band, represented by the $N$-dimensional vector $\mathbf{0}$, are employed as a data set D. Such dataset can therefore be seen as a collection of $N$ pairs $\left(\mathbf{x}_{i}, o_{i}\right)$, where $\mathbf{x}_{\boldsymbol{i}}$ denotes a sample vector of dimension $V$ (one volume of the fMRI time series) and $o_{i}$ the corresponding one-dimensional label (the EEG power oscillations in a specific band as extracted from the simultaneous recordings).

In machine learning for fMRI data analysis, it is common choice to use a linear model due to a typically high feature (i.e., voxel) to sample (i.e., scan) ratio. A standard linear model has the following form:

$o=y(\mathbf{x}, \mathbf{w})+\varepsilon$

where $y(\mathbf{x , w})$ is the deterministic input-output mapping part and $\varepsilon$ accounts for the noise in the measurements. The deterministic mapping can be modeled as [36]:

$y(\mathbf{x}, \mathbf{w})=\mathbf{w}^{\mathrm{T}} \phi(\mathbf{x})$

where $\phi: \mathfrak{R}^{V} \rightarrow \mathfrak{R}^{M}$ is a mapping from $V$-dimensional space of $\mathbf{x}$ into an $M$-dimensional one and $\mathbf{w}$ is an $M$-dimensional vector of parameters.

The aim of the estimation procedure is to find the "best" model parameters $\mathbf{w}$, evaluating the performances on an unknown dataset (generalization). One criterion could be to maximize the fit of the model to the training data, which leads to a least-squares solution. A perfect fit on the training 
dataset may not be optimal for generalization purposes, if the model is too complex, as in the case of highly dimensional multivariate patterns. Introducing some regularization to control for the smoothness of the estimate leads to the solution:

$\tilde{\mathbf{w}}=\left(\Phi^{\mathrm{T}} \Phi+\lambda I\right)^{-1} \Phi^{\mathrm{T}} \mathbf{o}$

which is sometimes called ridge regression solution. Regularization is particularly effective in training on small datasets (reducing the model complexity and subsequently the risk of overfitting), but one has to employ a suitable value for the weighting coefficient $\lambda$. One way to set this parameter is to perform cross-validation choosing the parameter that gives the highest generalization on the validation set(s).

\subsection{Relevance vector machine regression}

As an alternative to RR, kernel methods such as support vector machines (SVMs) [37] or RVMs can be used. Compared to SVM, RVM provides in many applications a much sparser model, typically an order of magnitude more compact, with little or no reduction of generalization error. Furthermore, no parameter has to be estimated in crossvalidation. For a complete description of RVM we refer to Refs. [26,38]. Here we will review briefly the concepts of RVM relevant in the context of multimodal imaging.

Without loss of generality, we refer to the first functional run as the training data set and subsequent runs as test set. Suppose that both the time courses of the voxels in the fMRI data and the simultaneously recorded EEG power oscillations have zero mean (note that the evaluation metric is based on Pearson correlation). The choice of a linear kernel results in the following model:

$y\left(\mathbf{X}_{1}, \mathbf{w}\right)=\mathbf{X}_{1} \mathbf{X}_{1}^{\mathrm{T}} \mathbf{w}=\mathbf{K} \mathbf{w}$

with $\mathbf{w}\left(n_{1} \times 1\right)$ being the model weights vector and $\mathbf{K}=\mathbf{X}_{1} \mathbf{X}_{1}^{\mathrm{T}}$ $\left(n_{1} \times n_{1}\right)$ the linear kernel constructed from the starting training dataset $\mathbf{X}_{1}\left(n_{1} \times V\right)$.

The RVM training aims at finding an estimate of the posterior distribution of the weights. This posterior distribution can then be used to perform predictions on a new dataset (second functional run). Denoting with $\tilde{\mathbf{w}}$ the estimated posterior mean, then the prediction on the second functional run $\mathbf{X}_{2}\left(n_{2} \times V\right)$ can be expressed as:

$\tilde{\mathbf{o}}_{2}=\mathbf{X}_{2} \mathbf{X}_{1}^{\mathrm{T}} \tilde{\mathbf{w}}$

It is possible to express the prediction in terms of maps:

$\tilde{\mathbf{0}}_{2}=\mathbf{X}_{2} \tilde{\mathbf{M}}$

with

$\tilde{\mathbf{M}}=\mathbf{X}_{1}^{\mathrm{T}} \tilde{\mathbf{w}}$

where $\tilde{\mathbf{M}}(V \times 1)$ can be interpreted as a map of relative contribution of the different voxels to the final prediction.

\section{Data}

Simultaneous EEG-fMRI data were collected from four healthy subjects (all males, mean age 32 years), three runs per subject. Each run was 5 min long and subjects were asked to close and open their eyes every $20 \mathrm{~s}$ indicated by an auditory cue.

\subsection{EEG Data}

EEG data were recorded using a 64-channel high-input impedance amplifier system specifically designed to operate in the MRI environment (Brainproducts, Munich, Germany). The setup consisted of two 32-channel MR plus amplifiers powered by a rechargeable power unit. The amplifiers were placed directly behind the scanner bore inside the MR room, which allowed the use of short wires with a total length of about $1.2 \mathrm{~m}$ from recording electrodes to the amplifier. Sintered $\mathrm{Ag} / \mathrm{AgCl}$ ring electrodes with built-in $5-\mathrm{k} \Omega$ resistors were used. Data were recorded from 62 equidistant scalp sites mounted in a cap system (Easycap, Falk Minow Services, Herrsching, Germany). Additional plastic electrode holders were tied into the cap at occipital scalp sites, which substantially improved subject comfort. Continuous data were also recorded from one electrode placed below the left eye to monitor eye blinks and another electrode placed at the lower back for electrocardiogram recording. All 64-channel data were referenced to the vertex. The data were recorded with a pass band of $0.016-250 \mathrm{~Hz}$ and digitized with 5000 samples/s at 16-bit resolution, resulting in a dynamic range of $16.38 \mathrm{mV}$. The amplified signal was transmitted via fiber-optic cables to a recording PC placed outside the MR room. Electrode impedances were maintained below $20 \mathrm{k} \Omega$ before recordings.

The EEG data processing was carried out using EEGLAB [39]. Functional MRI gradient and BCG artefacts were removed using the EEGLAB plug-in FMRIB 1.21 [32], as developed by the Centre for the Functional MRI of the Brain (Oxford, UK). For each subject, tICA was used to extract 62 components after concatenating the data from the three runs. The ICs were visually reviewed in order to discard components reflecting residual BCG or gradient artefacts [33].

For each subject, the component reflecting the highest modulation in the alpha power between the eyes open and eyes closed section was selected to create the EEG-based predictors. Time frequency decomposition (Morlet wavelets; window size $=1668 \mathrm{~ms}$ ) was applied to the IC time courses in order to extract the power modulations in the alpha band (8$14 \mathrm{~Hz}$ ). The power modulation over time of each separate run was convolved with a standard HRF [35] and resampled to the fMRI sampling rate $(0.5 \mathrm{~Hz}$, see below $)$ to obtain the final predictors.

For each subject, distributed source modeling was carried out using the EEG/MEG module of BrainVoyager QX v. 2.0 
(Brain Innovation, The Netherlands). A weighted minimum norm solution [28] was computed starting from the clean (after BCG correction) channel level data. Sources were placed on the vertices of the reconstructed cortex of each individual subject $[29,30]$. The sources exhibiting the highest modulation in the alpha band $(8-14 \mathrm{~Hz})$ were localized. A predictor for each run was obtained convolving the power modulation of the localized sources with a standard HRF.

\subsection{Functional MRI data}

Functional magnetic resonance time series were acquired in a 3-T system (Siemens Allegra, Erlangen, Germany). Functional runs consisted of 22 axial slices obtained with a T2*-weighted gradient echo, EPI sequence (TR $2 \mathrm{~s}$; TA 1.4 s; FOV $224 \times 224$; matrix size $64 \times 64$, voxel size $=3.5 \times 3.5 \times 4$ $\mathrm{mm})$. Anatomical images were obtained using a highresolution $(1 \times 1 \times 1 \mathrm{~mm})$ T1-weighted sequence.

After standard preprocessing steps (slice scan time correction; head motion correction; temporal filtering; resampling in three-dimensional standard, Talairach, space), the data sets of the three functional runs of each subject were used for the multivariate regression analysis using both RR and RVMs. Training was performed using the data of Runs 1 and 3, and predictions were evaluated on the data of Run 2. When using RR, the number of features/ voxels to be used was selected by means of cross-validation during the training phase using correlation-based ranking of the voxels.

Multivariate regression was compared to a conventional univariate regression analysis [general linear model (GLM)] and to an unsupervised multivariate analysis performed using ICA. GLM analysis was performed by concatenating Runs 1 and 3 and estimating voxels' beta weights for each EEG-related predictor. The estimated beta weights were then used to predict the EEG power oscillations in Run 2. The prediction was obtained by multiplying the voxel beta map estimated on Runs 1 and 3 with the fMRI data of Run 2. In order to predict EEG power oscillations using fMRI ICA analysis, the ICs with the highest correlation to each EEG-related predictor were identified in Runs 1 and 3. With the use of the similarity of the IC spatial maps [40], a candidate IC was selected to predict the EEG power oscillations in Run 2, and the correlation between the selected IC time course and the EEG power oscillations was used as a measure of prediction.

\section{Results}

Table 1 reports, for all subjects, the prediction results obtained using the same methods (RR, RVM, GLM and ICA) on the EEG-IC that mostly reflected the alpha power modulations induced during the eye open/closed periods. Accuracies are reported as the correlation between the real and predicted modulations in the second run (158 volumes; training on Runs 1 and 3). Multivariate regression methods
Table 1

Summary of the results obtained on the prediction of the EEG-IC characterizing the expected alpha modulation in Run 2 (training on Runs 1 and 3) for each subject and method

\begin{tabular}{lllll}
\hline & RR & RVM & GLM & ICA \\
\hline Subject 1 (FDM) & 0.88 & $\mathbf{0 . 9 0}$ & 0.69 & 0.85 \\
Subject 2 (FM) & 0.79 & $\mathbf{0 . 8 1}$ & 0.39 & 0.69 \\
Subject 3 (MR) & 0.68 & $\mathbf{0 . 7 4}$ & 0.36 & 0.22 \\
Subject 4 (TP) & 0.75 & $\mathbf{0 . 8 1}$ & 0.62 & 0.46 \\
\hline
\end{tabular}

Prediction accuracy is reported as the correlation between the predicted and the real alpha modulation in Run 2 (158 volumes; bold numbers highlight the highest accuracy for each subject).

(RR and RVM) outperform previously introduced univariate (GLM) and multivariate (ICA) approaches in all subjects, with a slight preference for RVM (highlighted).

Fig. 2 summarizes the results obtained in the prediction of the alpha power at the scalp level using RVM. Fig. 2A shows the group topography of the selected EEG-ICs together with the event-related spectral power plot (ERSP; computed on a time window of $-15,000$ to $15,000 \mathrm{~ms}$ relative to the closure of the eyes) showing the expected increase in alpha power when the eyes are closed. Fig. 2B shows the group map (after cortex-based alignment [41]) obtained from the training on Runs 1 and 3 of each subject, together with the group accuracy in the prediction of Run 2. Single-subject maps were sampled on the cortex, thresholded (in order to select the $50 \%$ most relevant vertices for the prediction) and binarized. A vertex in the group map is color coded if present in at least three of the four single-subject maps. The color in the map represents the sign of the correlation between the vertex time course and the alpha oscillations. The mean accuracy across subjects was computed after applying a Fisher $r$ to $z$ transform to the single-subject accuracies and then transforming back to $r$ the mean score. As expected, the mean map highlights several areas in the primary visual cortex, which are negatively correlated with the increase in alpha associated with closing the eyes. Interestingly, areas of the ventral visual system, the temporoparietal junction and anterior insula show a consistent positive correlation with the alpha modulation. Fig. 2C shows the real (black) and predicted (red) alpha oscillations at the level of the scalp for one exemplary subject (FDM) together with the subject's specific prediction accuracy.

Fig. 3 summarizes the results obtained in the prediction of the alpha power at the level of the cortical sources using RVM. Fig. 3A shows the group (after cortex-based alignment) source level distribution of activity highlighting the cortical areas that maximize the difference in alpha power between the eyes-open and eyes-closed events. The ERSP (computed on a time window of $-15,000$ to $15,000 \mathrm{~ms}$ relative to the closure of the eyes) of the highlighted cortical region is also reported and shows the expected increase in alpha power when the eyes are closed. Fig. 3B shows the group map (after cortex-based alignment) obtained from the training on Runs 1 and 3 of each subject, together with the 
A

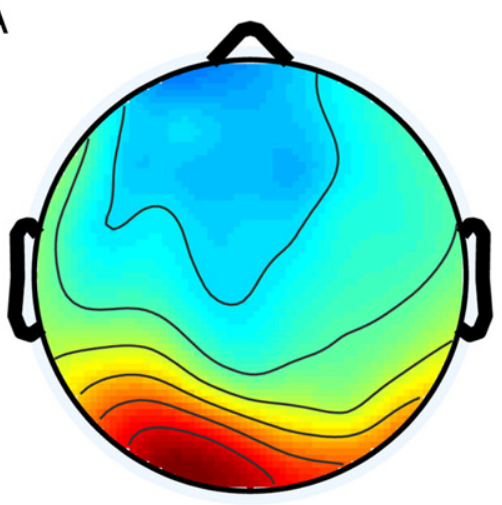

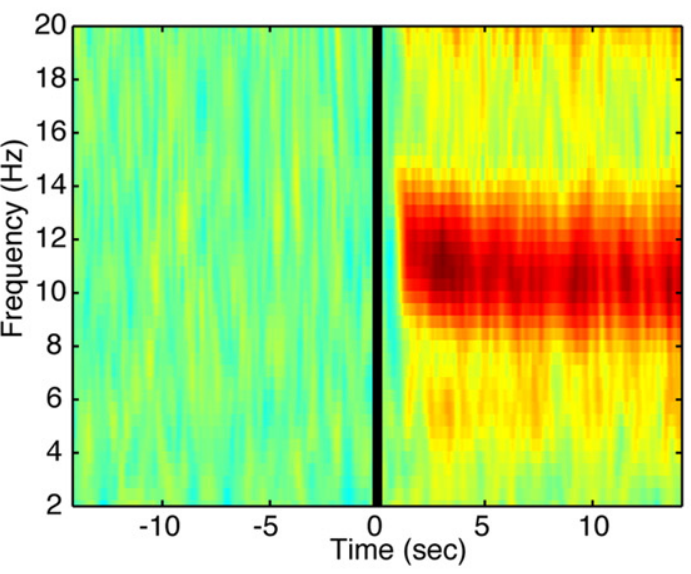

$r=0.82$

B

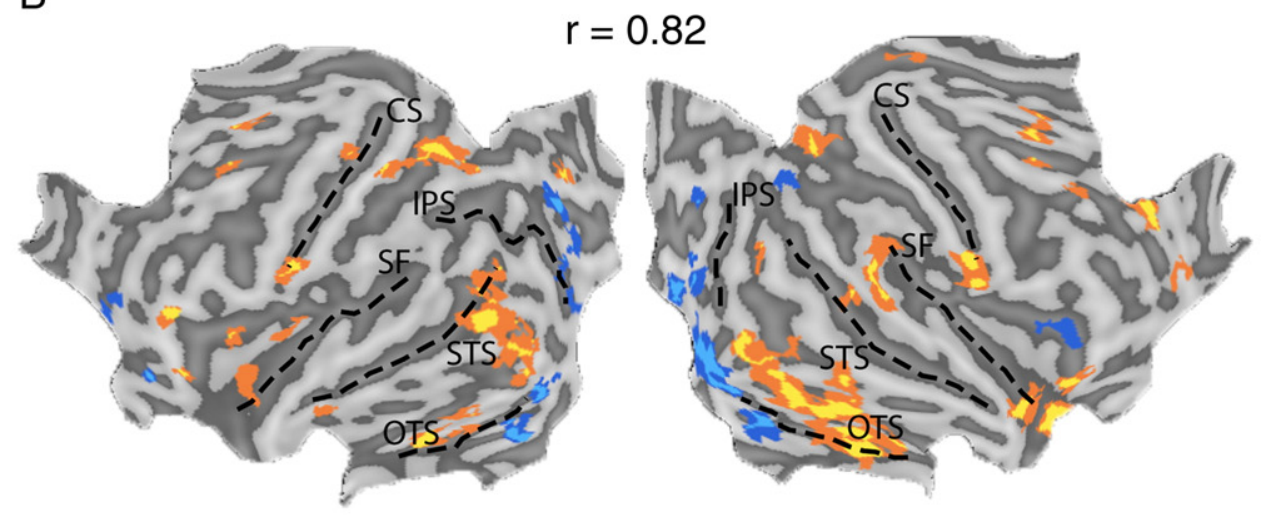

C $r=0.90$

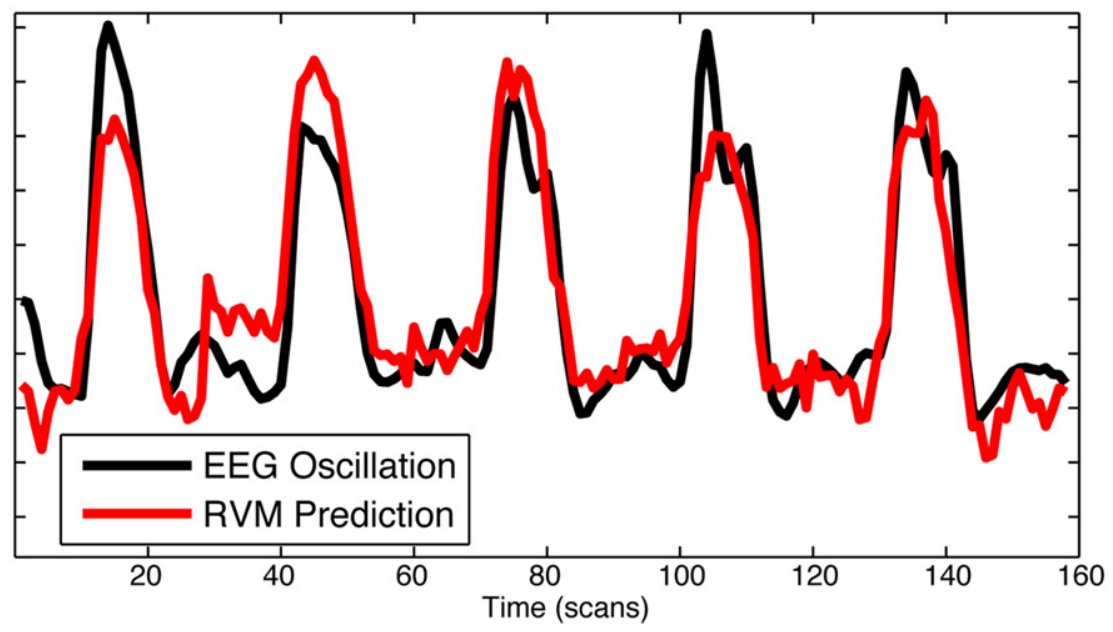

Fig. 2. Results obtained in the prediction of the alpha power at the scalp level using RVM. (A) Group topography of the selected EEG-ICs together with its ERSP. (B) Group map, projected on the mean flattened cortex of all subjects, obtained from the training of each subject. Single-subject maps were sampled on the cortex, thresholded (in order to select the 50\% most relevant vertices for the prediction) and binarized. A vertex in the group map is color coded if present in at least three of the four single-subject maps. The color in the map represents the sign of the correlation between the vertex time course and the alpha oscillations (red and yellow represent a positive correlation; blue and cyan represent a negative). The map is shown together with the group accuracy in the prediction of Run 2. Major sulci are highlighted in both hemispheres (central sulcus; inferior parietal sulcus; superior temporal sulcus; occipitotemporal sulcus; silvian fissure). (C) Comparison between real (black) and predicted (red) alpha oscillations at the level of the scalp for one exemplary subject (FDM) together with the subject's specific prediction accuracy.

group accuracy in the prediction of Run 2, as in Fig. 2B. The group map is shown together with the source level distribution of alpha activity (green transparent coding). A network of regions similar to Fig. 2 are highlighted as relevant for the prediction of the alpha source. Fig. $3 \mathrm{C}$ shows the proximity in the volume space between the localized 
A
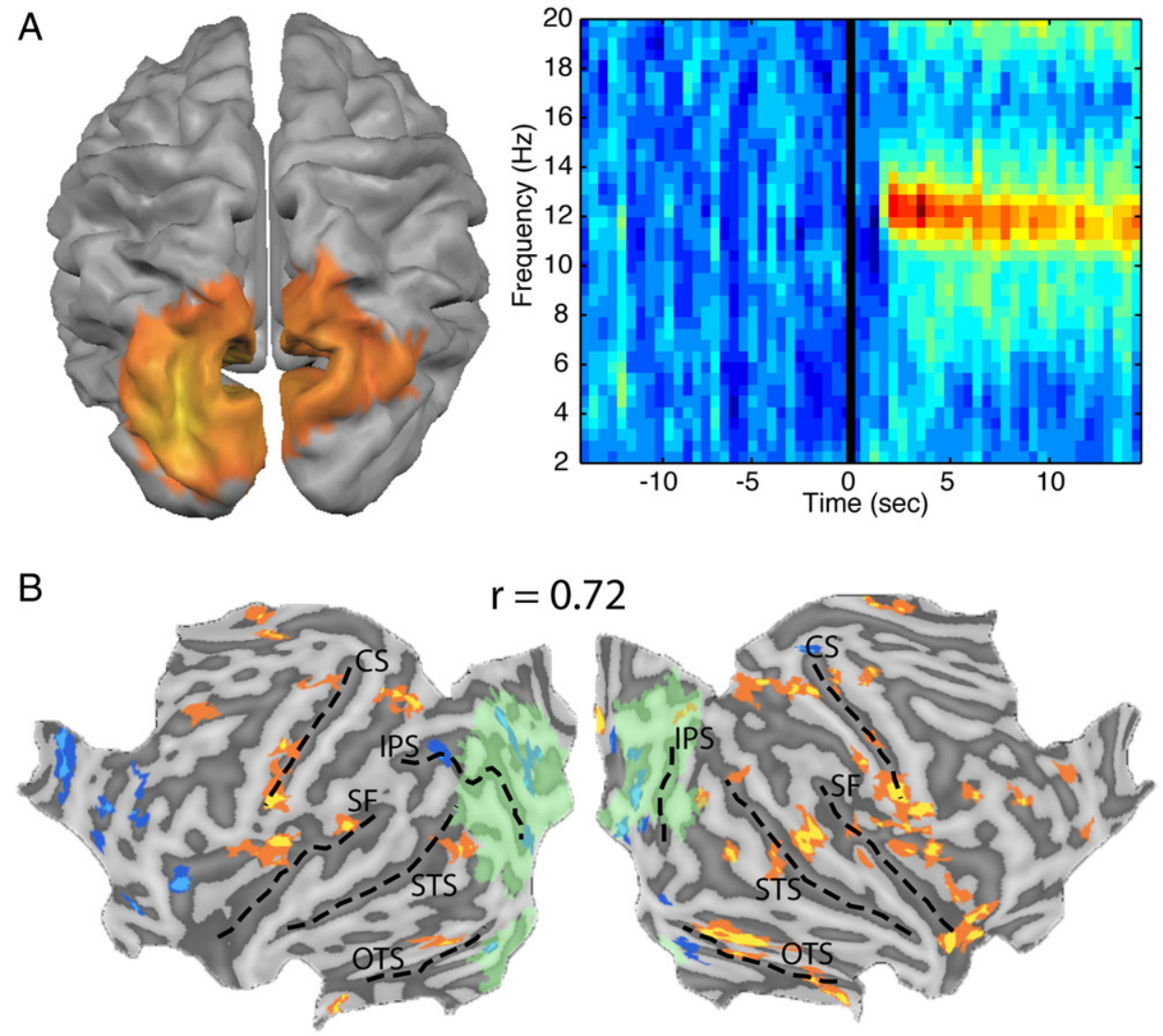

C

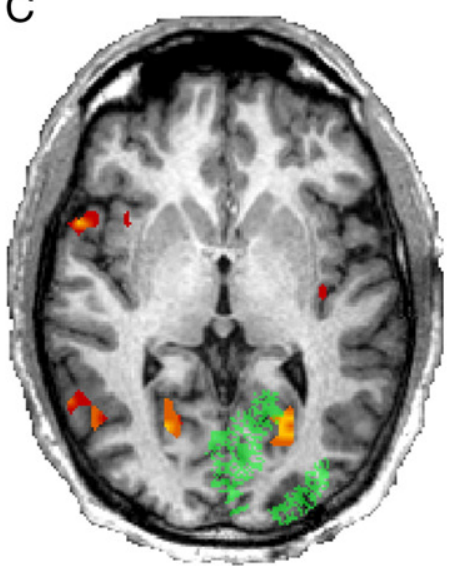

D

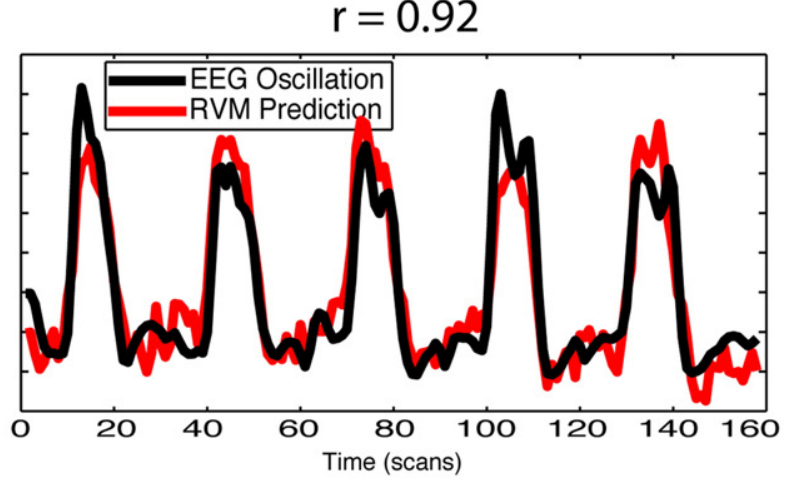

Fig. 3. Results obtained in the prediction of the alpha power at the level of the distributed cortical sources. (A) Group source level distribution highlighting the cortical areas that maximize the difference in alpha power between the eyes-open and eyes-closed events, together with its ERSP. (B) Group map, projected on the mean flattened cortex of all subjects, obtained from the training of each subject computed as in Fig. 2. The map is shown together with the source level distribution estimated from the EEG data (transparent coding) and the group accuracy in the prediction of Run 2. Major sulci are highlighted in both hemispheres (central sulcus; inferior parietal sulcus; superior temporal sulcus; occipito-temporal sulcus; silvian fissure). (C) Group fMRI map obtained from training and source level distribution estimated from the EEG data (green) sampled in the fMRI volume (one transversal slice). (D) Comparison between real (black) and predicted (red) alpha oscillations at the level of the scalp for one exemplary subject (FDM) together with the subject's specific prediction accuracy.

source of the alpha (green) and fMRI voxels relevant to the prediction of the alpha (red-yellow). Fig. 3D shows the real (black) and predicted (red) alpha oscillation at the level of the scalp for one exemplary subject (FDM) together with the subject-specific prediction accuracy.

\section{Discussion}

Multimodal imaging measurements allow studying brain dynamics with high temporal and spatial resolution. From an information-theoretic point of view [10], multimodal data have 
been shown to be more informative than the data from the single modalities. Simultaneous recordings of EEG and fMRI in particular have proven to be useful to study the nature of the coupling between "scalp" electrical activity and the BOLD signal in humans. The nature of the coupling between the two signals has been investigated making use of the correlation between single brain locations (univariate analysis) or global patterns (multivariate analysis) and the EEG signal.

Here we have introduced the use of multivariate regression and predictive modeling in multimodal imaging. Beyond the simple correlation between the two signals, we have shown that it is possible to predict, with considerable performances in an eyes-open/eyes-closed task, the electrical activity as measured by surface EEG from the multivariate patterns measured in fMRI. We have compared multivariate regression based on predictive modeling ( $R R$ and RVMs) techniques to previously introduced univariate (GLM) and multivariate (ICA) methods.

Conventional statistical analysis of combined EEG-fMRI measurements is based on the voxel-by-voxel hypothesis testing (GLM). The maps obtained with this procedure depict the voxels in the fMRI data that "best fit" the data-derived EEG power modulations and are estimated trying to minimize the fitting error in a least-square sense (LSE). Such explanatory procedure may be suboptimal when EEG power modulations are encoded in a multivariate and distributed fashion in the fMRI BOLD signal. Furthermore, the LSE estimation procedure of the GLM, while being optimal to explain the available data (training set), might fail when the purpose is to generalize the prediction to unseen data (test set).

Multivariate analysis techniques such as ICA have been previously used to analyze fMRI data sets from simultaneous EEG-fMRI recordings. Spatially ICs maps depict (distributed coherent fluctuations from) functionally connected brain regions and, like for the EEG power, are estimated in a datadriven way, without making use of the available experimental variables. However, the prediction ability of spatial ICA, evaluated post hoc as the correlation of a selected IC time course with the EEG power modulations, can only be considered as "incidental" as it is not at the basis of the estimation procedure.

Among multivariate techniques for fMRI, multivariate regression is an appealing tool for investigating the relevant question of the coupling between the fMRI time series and simultaneously acquired EEG oscillations. Given the massive multivariate nature of the dataset, the use of "regularized" models such as RR and RVMs is necessary in order to avoid "overfitting" of the data. These methods expressly aim at optimizing prediction abilities to unseen data sets, by estimating smooth models, which may reduce the fit of the training set. Thus, maps obtained using RR and RVM show the brain network that (multivariately) is most relevant in generalizing the learned EEG-fMRI coupling.

Our results show the superiority of RVM in predicting the alpha modulations induced by opening and closing the eyes consistently in four subjects. The mutual information between single-voxel fMRI responses and EEG signatures of the same responses has been proven to be relatively low [10]. Our results could be explained by better mutual information between multivariate fMRI patterns and the EEG signal. The highlighted predictive pattern in the fMRI data follows predictions based on previously reported results obtained in a similar design [15].

In previous applications the coupling between EEG and fMRI in humans has been measured at the level of "scalp" activity by means of single electrodes, group of electrodes or weighted averages of electrodes. Previous applications have discarded the investigation of the proximity between the sources of the electrical activity and the equivalent fMRI pattern. Distributed source analysis offers the possibility of analyzing both the EEG activity and the fMRI signal in the same space (i.e., the cortex) $[29,30]$. Here we have shown how distributed source analysis can be used to analyze the link between the BOLD signal and electrical activity at the level of estimated cortical sources within a multivariate framework accounting for the two modalities (fMRI and EEG). Our results show a slight decrease in the mean prediction across subjects (Figs. 2B and 3B) when moving from the channel space (scalp electrical activity) to the cortical space (source activity). This penalty may be easily explained by the fact that temporal ICA is applied to channellevel EEG and only one selected EEG-ICA component was used for the prediction. This approach has substantial denoising properties on the actual EEG data used in the prediction that are not exploited in the distributed source analysis. Conversely, all signal and noise EEG components are here projected from the channel to the source space with the express purpose of identifying regions of maximal EEG power modulation without the need of an explicit component selection. In future studies, the two approaches can be suitably combined by projecting channel EEG data reconstructed from only one or a few ICA components.

\section{Conclusions}

We have shown that multivariate regression is a valuable tool to study the coupling between the fMRI BOLD signal and the EEG power modulations, both at the level of the scalp and at the level of cortical sources. When comparing the predictions of different methods (GLM, ICA, RR, RVM) on unseen data of the eyes-open/eyes-closed protocol, we show that RVM-based prediction are more accurate than the other methods possibly due to the predictive (compared to explanatory or data-driven) and multivariate (compared to univariate) nature of the method. We suggest that highlighting the most relevant cortical patterns to the prediction of distributed cortical sources allows studying in more detail the coupling between the two signals and could help in improving the available source modeling algorithms.

Furthermore, our method would have a direct application in clinical settings (e.g., the localization of epileptic foci [42]) where simultaneous EEG-fMRI measurements and 
conventional statistical analysis are currently being used. We argue that the use of multivariate techniques in combination with cortical source modeling might improve the localization of aberrant electrical patterns (e.g., epileptic spikes).

\section{References}

[1] Dale AM, Halgren E. Spatiotemporal mapping of brain activity by integration of multiple imaging modalities. Curr Opin Neurobiol 2001;11:202-8.

[2] Valdes-Sosa PA, Sanchez-Bornot JM, Sotero JC, Iturria-Medina Y, Aleman-Gomez Y, Bosh-Bayard J, et al. Model driven EEG/fMRI fusion of brain images. Hum Brain Mapp 2009;30:2701-21.

[3] Goebel R, Esposito F. The added value of EEG-fMRI in imaging neuroscience. In: Mulert C, Lemieux L editors. EEG-fMRI physiology, technique and application. New York: Springer [in press].

[4] Ritter P, Villringer A. Simultaneous EEG-fMRI. Neurosci Behav Rev 2006;30:823-38.

[5] Debener S, Ullsperger M, Siegel M, Engel AK. Single-trial EEG-fMRI reveals the dynamics of cognitive function. Trends Cogn Sci 2006;10 (12):558-63.

[6] Logothetis NK. What we can do and what we cannot do with fMRI. Nature 2008;453:869-78.

[7] Sirotin YB, Aniruddha D. Anticipatory hemodynamic signals in sensory cortex not predicted by local neuronal activity. Nature 2009; 457:475-9.

[8] Logothetis NK, Pauls J, Augath M, Trinath T, Oeltermann A. Neurophysiological investigation of the basis of the fMRI signal. Nature 2001;412:150-7.

[9] Mukamel R, Gelbard H, Arieli A, Hasson U, Fried I, Malach R. Coupling between neuronal firing, field potentials, and fMRI in human auditory cortex. Science 2005;309:951-4.

[10] Ostwald D, Porcaro C, Bagshaw AP. An information theoretic approach to EEG-fMRI integration of visually evoked responses. NeuroImage 2010;49(1):498-516.

[11] Goldman RI, Stern JM, Engel Jr J, Cohen MS. Simultaneous EEG and fMRI of the alpha rhythm. NeuroReport 2002;13(18):2487-92.

[12] Laufs H, Kleinschmidt A, Beyerle A, Eger E, Salek-Haddadi A, Preibisch C, et al. EEG-correlated fMRI of human alpha activity. NeuroImage 2003;19(4):1463-76.

[13] Laufs H, Krakow K, Sterzer P, Eger E, Beyerle A, Salek-Haddadi A, et al. Electroencephalographic signatures of attentional and cognitive default modes in spontaneous brain activity fluctuations at rest. Proc Natl Acad Sci U S A 2003;100(19):11053-8.

[14] Moosmann M, Ritter P, Krastel I, Brink A, Thees S, Blankenburg F, et al. Correlates of alpha rhythm in functional magnetic resonance imaging and near infrared spectroscopy. NeuroImage 2003;20(1):145-58.

[15] Feige B, Scheffler K, Esposito F, Di Salle F, Hennig J, Seifritz E. Cortical and subcortical correlates of electroencephalographic alpha rhythm modulation. J Neurophysiol 2005;93(5):2864-72.

[16] Gonçalves SI, de Munck JC, Pouwels PJ, Schoonhoven R, Kuijer JP, Maurits NM, et al. Correlating the alpha rhythm to BOLD using simultaneous EEG/fMRI: inter-subject variability. NeuroImage 2005;30(1):203-13.

[17] Laufs H, Holt JL, Elfont R, Krams M, Paul JS, Krakow K, et al. Where the BOLD signal goes when alpha EEG leaves. NeuroImage 2006;31 (4):1408-18.

[18] de Munck JC, Gonçalves SI, Huijboom L, Kuijer JP, Pouwels PJ, Heethaar RM, et al. The hemodynamic response of the alpha rhythm: an EEG/fMRI study. NeuroImage 2007;35(3):1142-51.

[19] Giraud AL, Kleinschmidt A, Poeppel D, Lund TE, Frackowiak RS, Laufs H. Endogenous cortical rhythms determine cerebral specialization for speech perception and production. Neuron 2007;56(6):1127-34.

[20] Scheeringa R, Bastiaansen MC, Petersson KM, Oostenveld R, Norris DG, Hagoort P. Frontal theta EEG activity correlates negatively with the default mode network in resting state. Int J Psychophysiol 2008;67 (3):242-51.

[21] de Munck JC, Goncalves SI, Faes ThJC, Kuijer JPA, Powels PJW, Heethaar RM, et al. A study of the brain's resting state based on alpha band power, heart rate and fMRI. NeuroImage 2008;42:112-21.

[22] de Munck JC, Goncalves SI, Mammoliti R, Heethaar RM, Lopes da Silva FH. Interactions between different EEG frequency bands and their effect on alpha-fMRI correlations. NeuroImage 2009;47:69-76.

[23] Jann K, Dierks T, Boesch C, Kottlow M, Strik W, Koenig T. BOLD correlates of EEG alpha phase-locking and the fMRI default mode network. NeuroImage 2009;45:903-16.

[24] Mantini D, Perrucci MG, Del Gratta C, Romani GL, Corbetta M. Electrophysiological signatures of resting state networks in the human brain. Proc Natl Acad Sci U S A 2007;104(32):13170-5.

[25] Martínez-Montes E, Valdés-Sosa PA, Miwakeichi F, Goldman RI, Cohen MS. Concurrent EEG/fMRI analysis by multiway partial least squares. NeuroImage 2004;22(3):1023-34.

[26] Formisano E, De Martino F, Valente G. Multivariate analysis of fMRI time series: classification and regression of brain responses using machine learning. Magn Reson Imaging 2008;26(7):921-34.

[27] Friston K, Chu C, Mourão-Miranda J, Hulme O, Rees G, Penny W, et al. Bayesian decoding of brain images. NeuroImage 2008;39(1):181-205.

[28] Hamalainen M, Ilmoniemi RJ. Interpreting measured magnetic fields of the brain: estimates of current distributions. Technical Report TKKF-A559. Helsinki (Finland): Helsinki University of Technology; 1984.

[29] Esposito F, Aragri A, Piccoli T, Tedeschi G, Goebel R, Di Salle F. Distributed analysis of simultaneous EEG-fMRI time-series: modelling and interpretation issues. Magn Reson Imaging 2009;27:1120-30.

[30] Esposito F, Mulert C, Goebel R. Combined distributed source and single-trial EEG-fMRI modeling: application to effortful decision making processes. NeuroImage 2009;47(1):112-21.

[31] Allen PJ, Josephs O, Turner R. A method for removing imaging artifact from continuous EEG recorded during functional MRI. NeuroImage 2000;12(2):230-9.

[32] Niazy RK, Beckmann CF, Iannetti GD, Brady JM, Smith SM. Removal of FMRI environment artefacts from EEG data using optimal basis sets. NeuroImage 2005;28(3):720-37.

[33] Debener S, Strobel A, Sorger B, Peters J, Kranczioch C, Engel AK, et al. Improved quality of auditory event-related potentials recorded simultaneously with 3-T fMRI: removal of the ballistocardiogram artefact. NeuroImage 2007;34(2):587-97.

[34] Makeig S, Jung TP, Bell AJ, Ghahremani D, Sejnowski TJ. Blind separation of auditory event-related brain responses into independent components. Proc Natl Acad Sci U S A 1997;94(20):10979-84.

[35] Friston KJ, Fletcher P, Josephs O, Holmes A, Rugg MD, Turner R. Event-related fMRI: characterizing differential responses. NeuroImage 1998;7(1):30-40.

[36] Bishop CM. Pattern recognition and machine learning. New York: Springer; 2006.

[37] Vapnik VN. The nature of statistical learning theory. New York: Springer; 1995.

[38] Tipping ME. Sparse Bayesian learning and the relevance vector machine. J Mach Learn Res 2001;1:211-44.

[39] Delorme A, Makeig S. EEGLAB: an open source toolbox for analysis of single-trial EEG dynamics including independent component analysis. J Neurosci Methods 2004;134(1):9-21.

[40] Esposito F, Scarabino T, Hyvarinen A, Himberg J, Formisano E, Comani S, et al. Independent component analysis of fMRI group studies by self-organizing clustering. NeuroImage 2005;25(1):193-205.

[41] Goebel R, Esposito F, Formisano E. Analysis of functional image analysis contest (FIAC) data with BrainVoyager QX: from singlesubject to cortically aligned group general linear model analysis and self-organizing group independent component analysis. Hum Brain Mapp 2006;25(5):392-401.

[42] Vulliemoz S, Lemieux L, Daunizeau J, Michel CM, Duncan JS. The combination of EEG source imaging and EEG-correlated functional MRI to map epileptic networks. Epilepsia 2009:1-15. 